CRYSTALLOGRAPHIC COMMUNICATIONS

ISSN 2056-9890

\section{Crystal structure of pymetrozine}

Youngeun Jeon, Jineun Kim,* Gihaeng Kang and Tae Ho Kim*

Department of Chemistry and Research Institute of Natural Sciences, Gyeongsang National University, Jinju 660-701, Republic of Korea. *Correspondence e-mail:

thkim@gnu.ac.kr, jekim@gnu.ac.kr

Received 28 May 2015; accepted 4 June 2015

Edited by J. Simpson, University of Otago, New Zealand

The title compound, $\mathrm{C}_{10} \mathrm{H}_{11} \mathrm{~N}_{5} \mathrm{O}$ \{systematic name: 6-methyl4-[(E)-(pyridin-3-ylmethylidene)amino]-4,5-dihydro-1,2,4-triazin-3(2H)-one, $\mathrm{C}_{10} \mathrm{H}_{11} \mathrm{~N}_{5} \mathrm{O}$, is used as an antifeedant in pest control. The asymmetric unit comprises two independent molecules, $A$ and $B$, in which the dihedral angles between the pyridinyl and triazinyl ring planes [r.m.s. deviations $=0.0132$ and 0.0255$]$ are $11.60(6)$ and $18.06(4)^{\circ}$, respectively. In the crystal, $\mathrm{N}-\mathrm{H} \cdots \mathrm{O}, \mathrm{N}-\mathrm{H} \cdots \mathrm{N}, \mathrm{C}-\mathrm{H} \cdots \mathrm{N}$ and $\mathrm{C}-\mathrm{H} \cdots \mathrm{O}$ hydrogen bonds, together with weak $\pi-\pi$ interactions [ringcentroid separations $=3.5456(9)$ and $3.9142(9) \AA]$, link the pyridinyl and triazinyl rings of $A$ molecules, generating a three-dimensional network.

Keywords: crystal structure; pymetrozine; triazinone; insecticide; antifeedant; hydrogen bonding; $\pi-\pi$ interactions.

CCDC reference: 1404941

\section{Related literature}

For information on the toxicity and insecticidal properties of the title compound, see: He et al. (2011); Torres et al. (2003); Ausborn et al. (2005); Barati et al. (2013). For a related crystal structure, see: Wang et al. (2012).

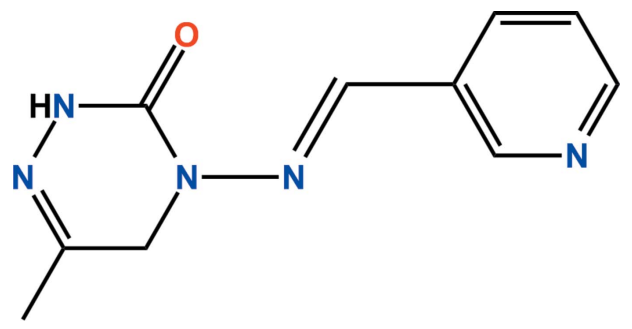

\section{Experimental}

2.1. Crystal data

$\mathrm{C}_{10} \mathrm{H}_{11} \mathrm{~N}_{5} \mathrm{O}$

$M_{r}=217.24$

Monoclinic, $P 2_{1} / n$

$a=8.0803(2) \mathrm{A}$

$b=23.7497(6) \AA$

$c=10.7846(3) \AA$

$\beta=98.962(1)^{\circ}$

$V=2044.35(9) \AA^{3}$

$Z=8$

Mo $K \alpha$ radiation

$\mu=0.10 \mathrm{~mm}^{-1}$

$T=173 \mathrm{~K}$

$0.37 \times 0.16 \times 0.09 \mathrm{~mm}$

\subsection{Data collection}

Bruker APEXII CCD diffractometer

Absorption correction: multi-scan (SADABS; Bruker, 2009)

$T_{\min }=0.964, T_{\max }=0.991$

\subsection{Refinement}

$R\left[F^{2}>2 \sigma\left(F^{2}\right)\right]=0.041$

$w R\left(F^{2}\right)=0.107$

$S=1.04$

3987 reflections

291 parameters

$\mathrm{H}$-atom parameters constrained

$\Delta \rho_{\max }=0.19{\mathrm{e} \AA^{-3}}^{-3}$

$\Delta \rho_{\min }=-0.31$ e $\AA^{-3}$

Table 1

Hydrogen-bond geometry $\left(\AA{ }^{\circ}\right)$.

\begin{tabular}{lllll}
\hline$D-\mathrm{H} \cdots A$ & $D-\mathrm{H}$ & $\mathrm{H} \cdots A$ & $D \cdots A$ & $D-\mathrm{H} \cdots A$ \\
\hline $\mathrm{N} 4-\mathrm{H} 4 \cdots \mathrm{O} 2^{\mathrm{i}}$ & 0.88 & 2.32 & $2.9545(17)$ & 129 \\
$\mathrm{~N} 4-\mathrm{H} 4 \cdots \mathrm{N}^{\mathrm{i}}$ & 0.88 & 2.43 & $3.2346(17)$ & 152 \\
$\mathrm{~N} 9-\mathrm{H} 9 \cdots \mathrm{N} 6{ }^{\text {ii }}$ & 0.88 & 2.04 & $2.882(2)$ & 159 \\
$\mathrm{C} 19-\mathrm{H} 19 A \cdots \mathrm{N} 1^{\mathrm{iii}}$ & 0.99 & 2.57 & $3.0852(19)$ & 112 \\
$\mathrm{C} 19-\mathrm{H} 19 A \cdots \mathrm{O} 1^{\text {iv }}$ & 0.99 & 2.60 & $3.5596(19)$ & 164 \\
$\mathrm{C} 20-\mathrm{H} 20 C \cdots \mathrm{O} 1^{\mathrm{v}}$ & 0.98 & 2.53 & $3.3906(19)$ & 147 \\
\hline
\end{tabular}

Symmetry codes: (i) $x-\frac{1}{2},-y+\frac{1}{2}, z+\frac{1}{2}$; (ii) $x, y, z-1$; (iii) $x-1, y, z-1$; (iv) $x-\frac{1}{2},-y+\frac{1}{2}, z-\frac{1}{2} ;(\mathrm{v})-x+\frac{1}{2}, y-\frac{1}{2},-z+\frac{1}{2}$.

Data collection: APEX2 (Bruker, 2009); cell refinement: SAINT (Bruker, 2009); data reduction: $S A I N T$; $\operatorname{program}(\mathrm{s})$ used to solve structure: SHELXTL (Sheldrick, 2008); program(s) used to refine structure: SHELXTL; molecular graphics: DIAMOND (Brandenburg, 2010); software used to prepare material for publication: SHELXTL.

\section{Acknowledgements}

This research was supported by the Basic Science Research Program through the National Research Foundation of Korea (NRF) funded by the Ministry of Education, Science and Technology (No. 2014R1A1A4A01009105).

Supporting information for this paper is available from the IUCr electronic archives (Reference: SJ5463). 


\section{data reports}

\section{References}

Ausborn, J., Wolf, H., Mader, W. \& Kayser, H. (2005). J. Exp. Biol. 208, 44514466.

Barati, R., Golmohammadi, G., Ghajarie, H., Zarabi, M. \& Mansouri, R. (2013). Pesticidi Fitomed. 28, 47-55.

Brandenburg, K. (2010). DIAMOND. Crystal Impact GbR, Bonn, Germany.

Bruker (2009). APEX2, SAINT and SADABS. Bruker AXS Inc., Madison, Wisconsin, USA.
He, Y., Chen, L., Chen, J., Zhang, J., Chen, L., Shen, J. \& Zhu, Y. C. (2011). Pest. Manag. Sci. 67, 483-491.

Sheldrick, G. M. (2008). Acta Cryst. A64, 112-122.

Torres, J. B., Silva-Torres, C. S. A. \& de Oliveira, J. V. (2003). Pesq. Agropec. Bras. 38, 459-466.

Wang, B., Ke, S., Kishore, B., Xu, X., Zou, Z. \& Li, Z. (2012). Synth. Commun. 42, 2327-2336. 


\section{supporting information}

Acta Cryst. (2015). E71, o461-o462［doi:10.1107/S2056989015010804]

\section{Crystal structure of pymetrozine}

\section{Youngeun Jeon, Jineun Kim, Gihaeng Kang and Tae Ho Kim}

\section{S1. Comment}

Pymetrozine: 4,5-dihydro-6-methyl-4-[(E)-(3-pyridinylmethylene)amino]-1,2,4-triazin-3(2H)-one, is an insecticide for the control of sucking insects, including the brown planthopper, Nilaparvata lugens, one of the most serious pests to affect rice crops (He et al., 2011). Its crystal structure is reported herein. In the title compound (Fig. 1), the asymmetric unit comprises two independent molecules (A and B) and the dihedral angles between the pyridinyl and triazinyl ring planes are 11.60 (6) and $18.06(4)^{\circ}$ for A and B, respectively. All bond lengths and bond angles are normal and comparable to those observed in a similar crystal structure (Wang et al., 2012).

The crystal structure, Fig. 2, is stabilized by intermolecular $\mathrm{N}-\mathrm{H} \cdots \mathrm{O}, \mathrm{N}-\mathrm{H} \cdots \mathrm{N}, \mathrm{C}-\mathrm{H} \cdots \mathrm{N}$ and $\mathrm{C}-\mathrm{H} \cdots \mathrm{O}$ hydrogen bonds (Table 1). In addition, weak intermolecular $\pi \cdots \pi$ interactions between the pyridinyl and triazinyl rings link adjacent A molecules [ $C g 1 \cdots C g 2^{2}, 3.5456(9) \AA$ and $C g 1 \cdots C g 2^{\mathrm{ii}}, 3.9142$ (9) $\left.\AA\right]$ symmetry codes: (i) $=x+1 / 2,-y+1 / 2, z+1 / 2$, (ii) $=x+1, y, z ; C g 1$ and $C g 2$ are the centroids of the $\mathrm{N} 1 \cdots \mathrm{C} 5$ and $\mathrm{N} 3 \cdots \mathrm{C} 9$ rings, respectively]. The $\pi \cdots \pi$ interactions together with $\mathrm{C} 1-\mathrm{H} 1 \cdots \mathrm{N} 5$ hydrogen bonds generate sheets of A molecules in the ac plane. All of these contacts combine to generate a three dimensional network, Fig. 2.

\section{S2. Experimental}

The title compound was purchased from the Dr. Ehrenstorfer $\mathrm{GmbH}$ Company. Slow evaporation of a solution in $\mathrm{CH}_{2} \mathrm{Cl}_{2}$ gave single crystals suitable for X-ray analysis.

\section{S3. Refinement}

All H-atoms were positioned geometrically and refined using a riding model with $\mathrm{d}(\mathrm{N}-\mathrm{H})=0.88 \AA, U_{\text {iso }}=1.2 U_{\text {eq }}(\mathrm{C})$ for the amine group, $\mathrm{d}(\mathrm{C}-\mathrm{H})=0.98 \AA, U_{\text {iso }}=1.2 U_{\text {eq }}(\mathrm{C})$ for the methyl group, $\mathrm{d}(\mathrm{C}-\mathrm{H})=0.99 \AA, U_{\text {iso }}=1.2 U_{\text {eq }}(\mathrm{C})$ for methylene $\mathrm{C}-\mathrm{H}$ and $\mathrm{d}(\mathrm{C}-\mathrm{H})=0.95 \AA, U_{\mathrm{iso}}=1.2 U_{\mathrm{eq}}(\mathrm{C})$ for aromatic $\mathrm{H}$ atoms 


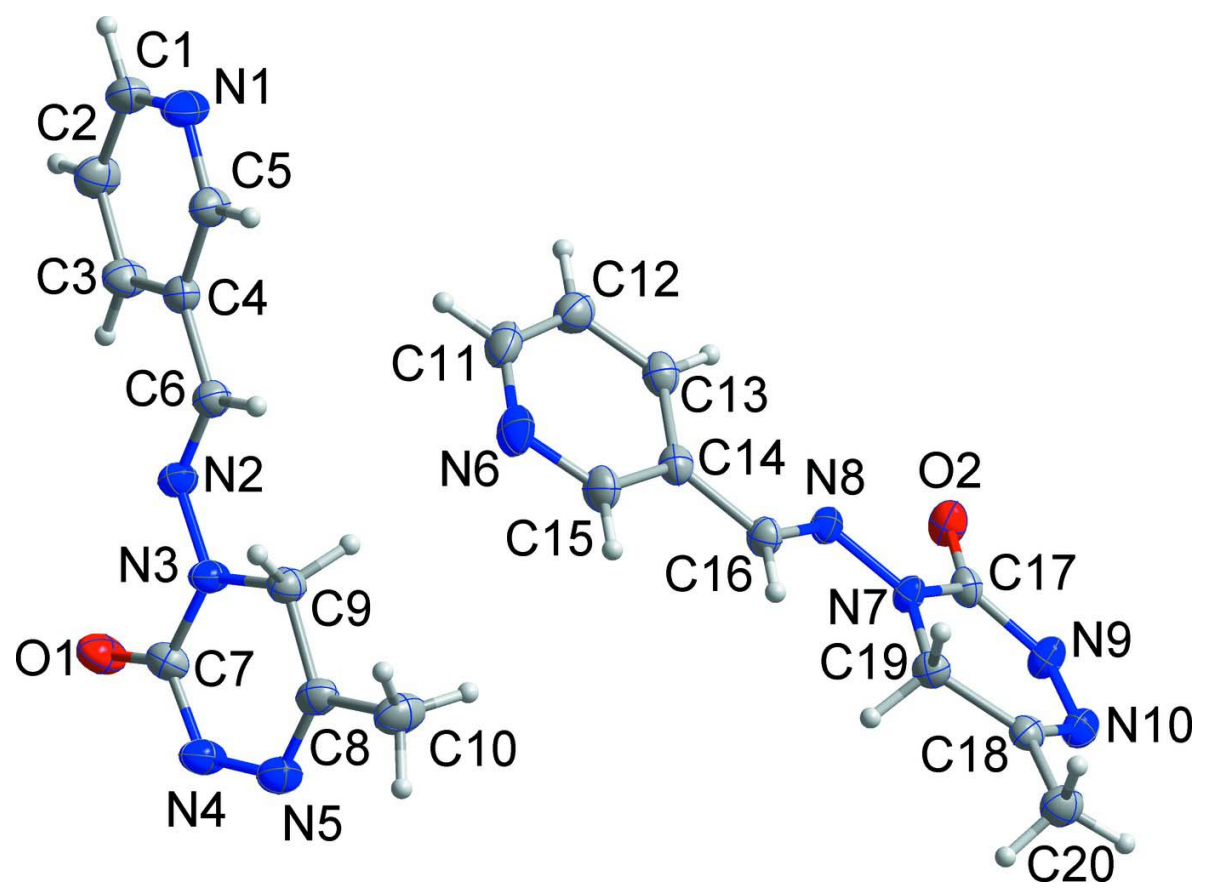

\section{Figure 1}

The asymmetric unit of the title compound with the atom-numbering scheme. Displacement ellipsoids are drawn at the $50 \%$ probability level. $\mathrm{H}$ atoms are shown as small spheres of arbitrary radius.

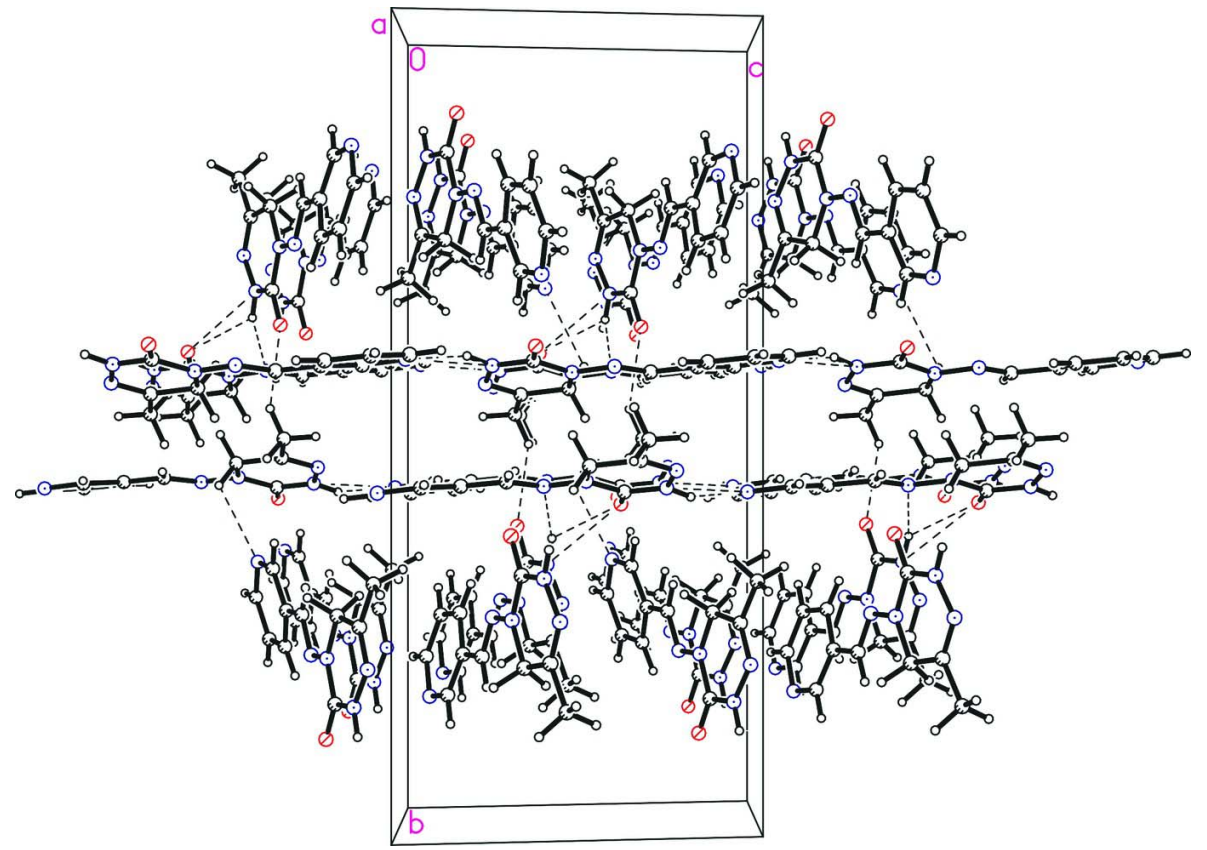

\section{Figure 2}

Crystal packing viewed along the $a$ axis. The hydrogen bonds are shown as dashed lines. 
6-Methyl-4-[(E)-(pyridin-3-ylmethylidene)amino]-4,5-dihydro-1,2,4-triazin-3(2H)-one

Crystal data

$\mathrm{C}_{10} \mathrm{H}_{11} \mathrm{~N}_{5} \mathrm{O}$

$M_{r}=217.24$

Monoclinic, $P 2_{1} / n$

$a=8.0803(2) \AA$

$b=23.7497$ (6) $\AA$

$c=10.7846(3) \AA$

$\beta=98.962(1)^{\circ}$

$V=2044.35(9) \AA^{3}$

$Z=8$

$F(000)=912$

$D_{\mathrm{x}}=1.412 \mathrm{Mg} \mathrm{m}^{-3}$

Mo $K \alpha$ radiation, $\lambda=0.71073 \AA$

Cell parameters from 3981 reflections

$\theta=2.7-27.3^{\circ}$

$\mu=0.10 \mathrm{~mm}^{-1}$

$T=173 \mathrm{~K}$

Block, colourless

$0.37 \times 0.16 \times 0.09 \mathrm{~mm}$

Data collection

Bruker APEXII CCD

diffractometer

Radiation source: fine-focus sealed tube

Graphite monochromator

$\varphi$ and $\omega$ scans

Absorption correction: multi-scan

(SADABS; Bruker, 2009)

$T_{\min }=0.964, T_{\max }=0.991$

19037 measured reflections

3987 independent reflections

3103 reflections with $I>2 \sigma(I)$

$R_{\text {int }}=0.042$

$\theta_{\max }=26.0^{\circ}, \theta_{\min }=2.1^{\circ}$

$h=-8 \rightarrow 9$

$k=-28 \rightarrow 29$

$l=-13 \rightarrow 12$

Refinement

Refinement on $F^{2}$

Least-squares matrix: full

$R\left[F^{2}>2 \sigma\left(F^{2}\right)\right]=0.041$

$w R\left(F^{2}\right)=0.107$

$S=1.04$

3987 reflections

291 parameters

0 restraints

Primary atom site location: structure-invariant

direct methods

Secondary atom site location: difference Fourier map

Hydrogen site location: inferred from neighbouring sites

$\mathrm{H}$-atom parameters constrained

$w=1 /\left[\sigma^{2}\left(F_{\mathrm{o}}{ }^{2}\right)+(0.0528 P)^{2}+0.3325 P\right]$

where $P=\left(F_{\mathrm{o}}^{2}+2 F_{\mathrm{c}}^{2}\right) / 3$

$(\Delta / \sigma)_{\max }=0.001$

$\Delta \rho_{\max }=0.19 \mathrm{e} \AA^{-3}$

$\Delta \rho_{\min }=-0.31 \mathrm{e} \AA^{-3}$

Special details

Geometry. All e.s.d.'s (except the e.s.d. in the dihedral angle between two 1.s. planes) are estimated using the full covariance matrix. The cell e.s.d.'s are taken into account individually in the estimation of e.s.d.'s in distances, angles and torsion angles; correlations between e.s.d.'s in cell parameters are only used when they are defined by crystal symmetry. An approximate (isotropic) treatment of cell e.s.d.'s is used for estimating e.s.d.'s involving 1.s. planes.

Refinement. Refinement of $F^{2}$ against ALL reflections. The weighted $R$-factor $w R$ and goodness of fit $S$ are based on $F^{2}$, conventional $R$-factors $R$ are based on $F$, with $F$ set to zero for negative $F^{2}$. The threshold expression of $F^{2}>\sigma\left(F^{2}\right)$ is used only for calculating $R$-factors(gt) etc. and is not relevant to the choice of reflections for refinement. $R$-factors based on $F^{2}$ are statistically about twice as large as those based on $F$, and $R$ - factors based on ALL data will be even larger.

Fractional atomic coordinates and isotropic or equivalent isotropic displacement parameters $\left(\AA^{2}\right)$

\begin{tabular}{lllll}
\hline & $x$ & $y$ & $z$ & $U_{\text {iso }} * / U_{\text {eq }}$ \\
\hline O1 & $0.54588(13)$ & $0.37396(4)$ & $0.67454(12)$ & $0.0371(3)$ \\
O2 & $0.65704(12)$ & $0.09515(4)$ & $-0.11461(10)$ & $0.0298(3)$ \\
N1 & $1.18740(16)$ & $0.17122(6)$ & $0.90565(15)$ & $0.0353(4)$ \\
N2 & $0.70889(14)$ & $0.27719(5)$ & $0.72493(13)$ & $0.0269(3)$ \\
N3 & $0.54303(15)$ & $0.27730(5)$ & $0.67218(13)$ & $0.0272(3)$
\end{tabular}




\begin{tabular}{|c|c|c|c|c|}
\hline N4 & $0.31072(15)$ & $0.32889(5)$ & $0.58809(14)$ & $0.0333(3)$ \\
\hline $\mathrm{H} 4$ & 0.2610 & 0.3618 & 0.5750 & $0.040^{*}$ \\
\hline N5 & $0.21533(15)$ & $0.28219(5)$ & $0.54719(14)$ & 0.0314 \\
\hline N6 & $0.56087(18)$ & $0.08007(6)$ & $0.54024(13)$ & $0.0362(3)$ \\
\hline N7 & $0.53681(14)$ & $0.07233(5)$ & $0.09543(12)$ & $0.0226(3)$ \\
\hline N8 & $0.43763(14)$ & $0.06556(5)$ & $-0.01909(12)$ & $0.0212(3)$ \\
\hline N9 & $0.41093(15)$ & $0.07374(5)$ & $-0.23457(12)$ & $0.0273(3)$ \\
\hline H9 & 0.4531 & 0.0850 & -0.3008 & $0.033^{*}$ \\
\hline N10 & $0.24931(16)$ & $0.05238(5)$ & $-0.25699(13)$ & $0.0266(3)$ \\
\hline $\mathrm{C} 1$ & $1.28109(19)$ & $0.21759(7)$ & 0.91859 (17) & $0.0325(4)$ \\
\hline H1 & 1.3940 & 0.2145 & 0.9587 & $0.039 *$ \\
\hline $\mathrm{C} 2$ & $1.22268(19)$ & $0.26979(7)$ & $0.87674(17)$ & $0.0346(4)$ \\
\hline $\mathrm{H} 2$ & 1.2949 & 0.3016 & 0.8865 & $0.041^{*}$ \\
\hline $\mathrm{C} 3$ & $1.05822(19)$ & $0.27537(6)$ & $0.82046(17)$ & 0.0319 (4) \\
\hline $\mathrm{H} 3$ & 1.0158 & 0.3109 & 0.7900 & $0.038^{*}$ \\
\hline $\mathrm{C} 4$ & $0.95585(18)$ & $0.22816(6)$ & $0.80914(15)$ & 0.0253 \\
\hline $\mathrm{C} 5$ & $1.02808(19)$ & $0.17746(6)$ & $0.85192(16)$ & $0.0305(4)$ \\
\hline H5 & 0.9594 & 0.1448 & 0.8423 & $0.037^{*}$ \\
\hline C6 & $0.77874(18)$ & $0.22966(6)$ & $0.75433(16)$ & 0.0269 (4) \\
\hline H6 & 0.7162 & 0.1958 & 0.7407 & $0.032 *$ \\
\hline $\mathrm{C} 7$ & $0.47257(18)$ & $0.32978(6)$ & $0.64618(16)$ & $0.0275(4)$ \\
\hline $\mathrm{C} 8$ & $0.28134(18)$ & $0.23414(7)$ & $0.57182(16)$ & $0.0281(4)$ \\
\hline C9 & $0.45481(18)$ & $0.22477(6)$ & $0.63922(16)$ & $0.0274(4)$ \\
\hline H9A & 0.4495 & 0.2029 & 0.7167 & $0.033^{*}$ \\
\hline H9B & 0.5183 & 0.2022 & 0.5853 & $0.033^{*}$ \\
\hline $\mathrm{C} 10$ & $0.1823(2)$ & $0.18265(7)$ & $0.53033(19)$ & $0.0397(5)$ \\
\hline $\mathrm{H} 10 \mathrm{~A}$ & 0.0639 & 0.1927 & 0.5073 & $0.060^{*}$ \\
\hline H10B & 0.1946 & 0.1552 & 0.5989 & $0.060^{*}$ \\
\hline $\mathrm{H} 10 \mathrm{C}$ & 0.2234 & 0.1663 & 0.4575 & $0.060^{*}$ \\
\hline C11 & $0.7273(2)$ & $0.08409(7)$ & $0.55839(17)$ & $0.0368(4)$ \\
\hline H11 & 0.7839 & 0.0886 & 0.6418 & $0.044 *$ \\
\hline $\mathrm{C} 12$ & $0.8219(2)$ & $0.08200(7)$ & $0.46212(17)$ & $0.0340(4)$ \\
\hline H12 & 0.9406 & 0.0842 & 0.4797 & $0.041^{*}$ \\
\hline $\mathrm{C} 13$ & $0.74117(19)$ & $0.07667(6)$ & $0.34017(16)$ & $0.0283(4)$ \\
\hline H13 & 0.8032 & 0.0761 & 0.2722 & $0.034 *$ \\
\hline $\mathrm{C} 14$ & $0.56786(18)$ & $0.07210(6)$ & $0.31837(15)$ & $0.0247(3)$ \\
\hline $\mathrm{C} 15$ & $0.4844(2)$ & $0.07337(7)$ & $0.42216(16)$ & $0.0315(4)$ \\
\hline H15 & 0.3661 & 0.0692 & 0.4080 & $0.038^{*}$ \\
\hline $\mathrm{C} 16$ & 0.46794 (19) & $0.06629(6)$ & $0.19390(15)$ & $0.0250(3)$ \\
\hline H16 & 0.3517 & 0.0580 & 0.1860 & $0.030^{*}$ \\
\hline $\mathrm{C} 17$ & $0.51141(17)$ & $0.07926(6)$ & $-0.12215(14)$ & 0.0219 \\
\hline $\mathrm{C} 18$ & $0.18317(18)$ & $0.03964(6)$ & $-0.16097(15)$ & $0.0241(3)$ \\
\hline C19 & 0.26507 (17) & $0.04665(6)$ & $-0.02804(15)$ & $0.0233(3)$ \\
\hline H19A & 0.2010 & 0.0744 & 0.0140 & $0.028^{*}$ \\
\hline H19B & 0.2626 & 0.0103 & 0.0164 & $0.028^{*}$ \\
\hline $\mathrm{C} 20$ & $0.01097(18)$ & $0.01533(7)$ & $-0.17992(17)$ & $0.0324(4)$ \\
\hline $\mathrm{H} 20 \mathrm{~A}$ & -0.0309 & 0.0125 & -0.2700 & $0.049^{*}$ \\
\hline H20B & -0.0633 & 0.0397 & -0.1402 & $0.049 *$ \\
\hline
\end{tabular}


supporting information

\begin{tabular}{|c|c|c|c|c|c|c|}
\hline $\mathrm{O} 1$ & $00212(6)$ & $00260(6)$ & $00533(9)$ & $00021(5)$ & $00041(5)$ & $-00001(6)$ \\
\hline $\mathrm{O} 2$ & $0.0280(6)$ & $0.0374(6)$ & $0.0254(7)$ & $-0.0084(4)$ & $0.0079(5)$ & $-0.0027(5)$ \\
\hline N1 & $0.0292(7)$ & $0.0321(7)$ & $0.0420(10)$ & $0.0044(6)$ & $-0.0023(6)$ & $0.0034(7)$ \\
\hline N2 & $0.0211(7)$ & $0.0294(7)$ & $0.0295(8)$ & $0.0016(5)$ & $0.0015(5)$ & $0.0001(6)$ \\
\hline N3 & $0.0219(6)$ & $0.0254(7)$ & $0.0327(8)$ & $0.0026(5)$ & $-0.0004(6)$ & $0.0018(6)$ \\
\hline N4 & $0.0254(7)$ & $0.0264(7)$ & $0.0465(10)$ & $0.0062(5)$ & $0.0010(6)$ & $0.0034(7)$ \\
\hline N5 & $0.0243(7)$ & $0.0336(7)$ & $0.0354(9)$ & $0.0011(5)$ & $0.0024(6)$ & $0.0027(6)$ \\
\hline N6 & $0.0471(9)$ & $0.0417(8)$ & $0.0204(8)$ & $-0.0003(6)$ & $0.0073(6)$ & $-0.0018(7)$ \\
\hline N7 & $0.0266(7)$ & $0.0222(6)$ & $0.0185(7)$ & $-0.0007(5)$ & $0.0024(5)$ & $-0.0007(6)$ \\
\hline N8 & $0.0214(6)$ & $0.0250(6)$ & $0.0170(7)$ & $-0.0028(5)$ & $0.0029(5)$ & $0.0000(5)$ \\
\hline N9 & $0.0299(7)$ & $0.0342(7)$ & $0.0182(7)$ & $-0.0078(5)$ & $0.0045(5)$ & $0.0019(6)$ \\
\hline N10 & $0.0275(7)$ & $0.0257(7)$ & $0.0256(8)$ & $-0.0025(5)$ & $0.0004(6)$ & $-0.0010(6)$ \\
\hline $\mathrm{C} 1$ & $0.0243(8)$ & $0.0389(9)$ & $0.0330(10)$ & $0.0029(7)$ & $0.0007(7)$ & -0.0018 \\
\hline $\mathrm{C} 2$ & $0.0274(8)$ & $0.0322(9)$ & $0.0431(12)$ & $-0.0046(6)$ & $0.0024(7)$ & $-0.0014(8)$ \\
\hline $\mathrm{C} 3$ & $0.0310(9)$ & $0.0248(8)$ & $0.0392(11)$ & $0.0023(6)$ & $0.0031(7)$ & $0.0020(8)$ \\
\hline $\mathrm{C} 4$ & $0.0252(8)$ & $0.0270(8)$ & $0.0236(9)$ & $0.0028(6)$ & $0.0037(6)$ & $-0.0019(7)$ \\
\hline $\mathrm{C} 5$ & $0.0291(8)$ & $0.0256(8)$ & $0.0352(10)$ & $-0.0008(6)$ & $0.0002(7)$ & $-0.0008(7)$ \\
\hline C6 & $0.0260(8)$ & $0.0264(8)$ & $0.0278(9)$ & $0.0006(6)$ & $0.0031(7)$ & $-0.0001(7)$ \\
\hline $\mathrm{C} 7$ & $0.0257(8)$ & $0.0265(8)$ & $0.0313(10)$ & $0.0039(6)$ & $0.0080(7)$ & $0.0031(7)$ \\
\hline $\mathrm{C} 8$ & $0.0246(8)$ & $0.0332(8)$ & $0.0267(9)$ & $0.0011(6)$ & $0.0049(7)$ & $0.0029(7)$ \\
\hline C9 & $0.0271(8)$ & $0.0246(8)$ & $0.0299(10)$ & $0.0011(6)$ & $0.0022(7)$ & $0.0017(7)$ \\
\hline $\mathrm{C} 10$ & $0.0313(9)$ & $0.0361(9)$ & $0.0482(12)$ & $-0.0030(7)$ & $-0.0046(8)$ & $0.0022(9)$ \\
\hline C11 & $0.0496(11)$ & $0.0346(9)$ & $0.0233(10)$ & $0.0021(8)$ & $-0.0032(8)$ & $-0.0025(8)$ \\
\hline $\mathrm{C} 12$ & $0.0341(9)$ & $0.0313(9)$ & $0.0344(11)$ & $-0.0002(7)$ & $-0.0015(7)$ & $-0.0027(8)$ \\
\hline $\mathrm{C} 13$ & $0.0357(9)$ & $0.0231(8)$ & $0.0270(9)$ & $-0.0011(6)$ & $0.0078(7)$ & $-0.0009(7)$ \\
\hline C14 & $0.0320(8)$ & $0.0206(7)$ & $0.0219(9)$ & $-0.0001(6)$ & $0.0052(6)$ & $-0.0004(7)$ \\
\hline $\mathrm{C} 15$ & $0.0358(9)$ & $0.0349(9)$ & $0.0249(9)$ & $-0.0010(7)$ & $0.0079(7)$ & -0.0005 \\
\hline $\mathrm{C} 16$ & $0.0270(8)$ & $0.0268(8)$ & $0.0221(9)$ & $-0.0006(6)$ & $0.0064(6)$ & -0.0003 \\
\hline $\mathrm{C} 17$ & $0.0268(8)$ & $0.0190(7)$ & $0.0207(8)$ & $-0.0017(6)$ & $0.0067(6)$ & $-0.0010(6)$ \\
\hline $\mathrm{C} 18$ & $0.0257(8)$ & $0.0194(7)$ & $0.0267(9)$ & $0.0016(6)$ & $0.0023(7)$ & $-0.0004(7)$ \\
\hline C19 & $0.0223(7)$ & $0.0240(7)$ & $0.0243(9)$ & $-0.0006(5)$ & $0.0059(6)$ & $0.0024(7)$ \\
\hline $\mathrm{C} 20$ & $0.0268(8)$ & $0.0328(8)$ & $0.0367(10)$ & $-0.0015(6)$ & $0.0021(7)$ & $0.0004(8)$ \\
\hline
\end{tabular}

Geometric parameters $\left(\hat{A},{ }^{o}\right)$

\begin{tabular}{llll}
\hline $\mathrm{O} 1-\mathrm{C} 7$ & $1.2202(18)$ & $\mathrm{C} 4-\mathrm{C} 5$ & $1.385(2)$ \\
$\mathrm{O} 2-\mathrm{C} 17$ & $1.2263(16)$ & $\mathrm{C} 4-\mathrm{C} 6$ & $1.461(2)$ \\
$\mathrm{N} 1-\mathrm{C} 1$ & $1.331(2)$ & $\mathrm{C} 5-\mathrm{H} 5$ & 0.9500 \\
$\mathrm{~N} 1-\mathrm{C} 5$ & $1.3352(19)$ & $\mathrm{C} 6-\mathrm{H} 6$ & 0.9500 \\
$\mathrm{~N} 2-\mathrm{C} 6$ & $1.2797(19)$ & $\mathrm{C} 8-\mathrm{C} 10$ & $1.491(2)$ \\
$\mathrm{N} 2-\mathrm{N} 3$ & $1.3719(17)$ & $\mathrm{C} 8-\mathrm{C} 9$ & $1.492(2)$ \\
$\mathrm{N} 3-\mathrm{C} 7$ & $1.3807(18)$ & $\mathrm{C} 9-\mathrm{H} 9 \mathrm{~A}$ & 0.9900 \\
$\mathrm{~N} 3-\mathrm{C} 9$ & $1.4534(18)$ & $\mathrm{C} 9-\mathrm{H} 9 \mathrm{~B}$ & 0.9900
\end{tabular}




\begin{tabular}{|c|c|c|c|}
\hline N4-C7 & $1.3594(19)$ & $\mathrm{C} 10-\mathrm{H} 10 \mathrm{~A}$ & 0.9800 \\
\hline $\mathrm{N} 4-\mathrm{N} 5$ & $1.3832(17)$ & $\mathrm{C} 10-\mathrm{H} 10 \mathrm{~B}$ & 0.9800 \\
\hline $\mathrm{N} 4-\mathrm{H} 4$ & 0.8800 & $\mathrm{C} 10-\mathrm{H} 10 \mathrm{C}$ & 0.9800 \\
\hline $\mathrm{N} 5-\mathrm{C} 8$ & $1.2701(19)$ & $\mathrm{C} 11-\mathrm{C} 12$ & $1.383(2)$ \\
\hline $\mathrm{N} 6-\mathrm{C} 11$ & $1.332(2)$ & $\mathrm{C} 11-\mathrm{H} 11$ & 0.9500 \\
\hline $\mathrm{N} 6-\mathrm{C} 15$ & $1.335(2)$ & $\mathrm{C} 12-\mathrm{C} 13$ & $1.380(2)$ \\
\hline $\mathrm{N} 7-\mathrm{C} 16$ & $1.281(2)$ & $\mathrm{C} 12-\mathrm{H} 12$ & 0.9500 \\
\hline $\mathrm{N} 7-\mathrm{N} 8$ & $1.3730(17)$ & $\mathrm{C} 13-\mathrm{C} 14$ & $1.388(2)$ \\
\hline $\mathrm{N} 8-\mathrm{C} 17$ & $1.3795(19)$ & $\mathrm{C} 13-\mathrm{H} 13$ & 0.9500 \\
\hline $\mathrm{N} 8-\mathrm{C} 19$ & $1.4536(17)$ & $\mathrm{C} 14-\mathrm{C} 15$ & $1.394(2)$ \\
\hline N9- $-\mathrm{C} 17$ & $1.3564(19)$ & $\mathrm{C} 14-\mathrm{C} 16$ & $1.461(2)$ \\
\hline N9-N10 & $1.3867(17)$ & $\mathrm{C} 15-\mathrm{H} 15$ & 0.9500 \\
\hline N9- $\mathrm{H} 9$ & 0.8800 & $\mathrm{C} 16-\mathrm{H} 16$ & 0.9500 \\
\hline $\mathrm{N} 10-\mathrm{C} 18$ & $1.274(2)$ & $\mathrm{C} 18-\mathrm{C} 20$ & $1.491(2)$ \\
\hline $\mathrm{C} 1-\mathrm{C} 2$ & $1.377(2)$ & $\mathrm{C} 18-\mathrm{C} 19$ & $1.492(2)$ \\
\hline $\mathrm{C} 1-\mathrm{H} 1$ & 0.9500 & C19-H19A & 0.9900 \\
\hline $\mathrm{C} 2-\mathrm{C} 3$ & $1.379(2)$ & $\mathrm{C} 19-\mathrm{H} 19 \mathrm{~B}$ & 0.9900 \\
\hline $\mathrm{C} 2-\mathrm{H} 2$ & 0.9500 & $\mathrm{C} 20-\mathrm{H} 20 \mathrm{~A}$ & 0.9800 \\
\hline $\mathrm{C} 3-\mathrm{C} 4$ & $1.387(2)$ & $\mathrm{C} 20-\mathrm{H} 20 \mathrm{~B}$ & 0.9800 \\
\hline $\mathrm{C} 3-\mathrm{H} 3$ & 0.9500 & $\mathrm{C} 20-\mathrm{H} 20 \mathrm{C}$ & 0.9800 \\
\hline $\mathrm{C} 1-\mathrm{N} 1-\mathrm{C} 5$ & $116.61(13)$ & $\mathrm{N} 3-\mathrm{C} 9-\mathrm{H} 9 \mathrm{~B}$ & 109.2 \\
\hline $\mathrm{C} 6-\mathrm{N} 2-\mathrm{N} 3$ & $117.97(12)$ & $\mathrm{C} 8-\mathrm{C} 9-\mathrm{H} 9 \mathrm{~B}$ & 109.2 \\
\hline $\mathrm{N} 2-\mathrm{N} 3-\mathrm{C} 7$ & $115.55(12)$ & $\mathrm{H} 9 \mathrm{~A}-\mathrm{C} 9-\mathrm{H} 9 \mathrm{~B}$ & 107.9 \\
\hline $\mathrm{N} 2-\mathrm{N} 3-\mathrm{C} 9$ & $120.66(11)$ & $\mathrm{C} 8-\mathrm{C} 10-\mathrm{H} 10 \mathrm{~A}$ & 109.5 \\
\hline $\mathrm{C} 7-\mathrm{N} 3-\mathrm{C} 9$ & $123.66(12)$ & $\mathrm{C} 8-\mathrm{C} 10-\mathrm{H} 10 \mathrm{~B}$ & 109.5 \\
\hline $\mathrm{C} 7-\mathrm{N} 4-\mathrm{N} 5$ & $127.42(12)$ & $\mathrm{H} 10 \mathrm{~A}-\mathrm{C} 10-\mathrm{H} 10 \mathrm{~B}$ & 109.5 \\
\hline $\mathrm{C} 7-\mathrm{N} 4-\mathrm{H} 4$ & 116.3 & $\mathrm{C} 8-\mathrm{C} 10-\mathrm{H} 10 \mathrm{C}$ & 109.5 \\
\hline $\mathrm{N} 5-\mathrm{N} 4-\mathrm{H} 4$ & 116.3 & $\mathrm{H} 10 \mathrm{~A}-\mathrm{C} 10-\mathrm{H} 10 \mathrm{C}$ & 109.5 \\
\hline $\mathrm{C} 8-\mathrm{N} 5-\mathrm{N} 4$ & $117.31(13)$ & $\mathrm{H} 10 \mathrm{~B}-\mathrm{C} 10-\mathrm{H} 10 \mathrm{C}$ & 109.5 \\
\hline $\mathrm{C} 11-\mathrm{N} 6-\mathrm{C} 15$ & $117.07(15)$ & $\mathrm{N} 6-\mathrm{C} 11-\mathrm{C} 12$ & $123.42(16)$ \\
\hline $\mathrm{C} 16-\mathrm{N} 7-\mathrm{N} 8$ & $117.67(12)$ & N6-C11-H11 & 118.3 \\
\hline $\mathrm{N} 7-\mathrm{N} 8-\mathrm{C} 17$ & $115.60(11)$ & $\mathrm{C} 12-\mathrm{C} 11-\mathrm{H} 11$ & 118.3 \\
\hline $\mathrm{N} 7-\mathrm{N} 8-\mathrm{C} 19$ & $121.02(12)$ & $\mathrm{C} 13-\mathrm{C} 12-\mathrm{C} 11$ & $118.97(16)$ \\
\hline $\mathrm{C} 17-\mathrm{N} 8-\mathrm{C} 19$ & $123.36(12)$ & $\mathrm{C} 13-\mathrm{C} 12-\mathrm{H} 12$ & 120.5 \\
\hline $\mathrm{C} 17-\mathrm{N} 9-\mathrm{N} 10$ & $127.41(13)$ & $\mathrm{C} 11-\mathrm{C} 12-\mathrm{H} 12$ & 120.5 \\
\hline $\mathrm{C} 17-\mathrm{N} 9-\mathrm{H} 9$ & 116.3 & $\mathrm{C} 12-\mathrm{C} 13-\mathrm{C} 14$ & $118.91(16)$ \\
\hline N10-N9- $\mathrm{H} 9$ & 116.3 & $\mathrm{C} 12-\mathrm{C} 13-\mathrm{H} 13$ & 120.5 \\
\hline $\mathrm{C} 18-\mathrm{N} 10-\mathrm{N} 9$ & $116.63(13)$ & $\mathrm{C} 14-\mathrm{C} 13-\mathrm{H} 13$ & 120.5 \\
\hline $\mathrm{N} 1-\mathrm{C} 1-\mathrm{C} 2$ & $123.36(14)$ & $\mathrm{C} 13-\mathrm{C} 14-\mathrm{C} 15$ & $117.63(15)$ \\
\hline $\mathrm{N} 1-\mathrm{C} 1-\mathrm{H} 1$ & 118.3 & $\mathrm{C} 13-\mathrm{C} 14-\mathrm{C} 16$ & $124.17(15)$ \\
\hline $\mathrm{C} 2-\mathrm{C} 1-\mathrm{H} 1$ & 118.3 & $\mathrm{C} 15-\mathrm{C} 14-\mathrm{C} 16$ & $118.20(14)$ \\
\hline $\mathrm{C} 1-\mathrm{C} 2-\mathrm{C} 3$ & $119.24(15)$ & $\mathrm{N} 6-\mathrm{C} 15-\mathrm{C} 14$ & $123.96(15)$ \\
\hline $\mathrm{C} 1-\mathrm{C} 2-\mathrm{H} 2$ & 120.4 & $\mathrm{~N} 6-\mathrm{C} 15-\mathrm{H} 15$ & 118.0 \\
\hline $\mathrm{C} 3-\mathrm{C} 2-\mathrm{H} 2$ & 120.4 & $\mathrm{C} 14-\mathrm{C} 15-\mathrm{H} 15$ & 118.0 \\
\hline $\mathrm{C} 2-\mathrm{C} 3-\mathrm{C} 4$ & $118.79(14)$ & $\mathrm{N} 7-\mathrm{C} 16-\mathrm{C} 14$ & $120.11(14)$ \\
\hline $\mathrm{C} 2-\mathrm{C} 3-\mathrm{H} 3$ & 120.6 & $\mathrm{~N} 7-\mathrm{C} 16-\mathrm{H} 16$ & 119.9 \\
\hline $\mathrm{C} 4-\mathrm{C} 3-\mathrm{H} 3$ & 120.6 & $\mathrm{C} 14-\mathrm{C} 16-\mathrm{H} 16$ & 119.9 \\
\hline
\end{tabular}




\begin{tabular}{|c|c|}
\hline $\mathrm{C} 5-\mathrm{C} 4-\mathrm{C} 3$ & $117.30(14)$ \\
\hline $\mathrm{C} 5-\mathrm{C} 4-\mathrm{C} 6$ & $119.41(13)$ \\
\hline $\mathrm{C} 3-\mathrm{C} 4-\mathrm{C} 6$ & $123.28(13)$ \\
\hline $\mathrm{N} 1-\mathrm{C} 5-\mathrm{C} 4$ & $124.65(14)$ \\
\hline $\mathrm{N} 1-\mathrm{C} 5-\mathrm{H} 5$ & 117.7 \\
\hline $\mathrm{C} 4-\mathrm{C} 5-\mathrm{H} 5$ & 117.7 \\
\hline $\mathrm{N} 2-\mathrm{C} 6-\mathrm{C} 4$ & $119.22(14)$ \\
\hline $\mathrm{N} 2-\mathrm{C} 6-\mathrm{H} 6$ & 120.4 \\
\hline $\mathrm{C} 4-\mathrm{C} 6-\mathrm{H} 6$ & 120.4 \\
\hline $\mathrm{O} 1-\mathrm{C} 7-\mathrm{N} 4$ & $121.57(14)$ \\
\hline $\mathrm{O} 1-\mathrm{C} 7-\mathrm{N} 3$ & $123.81(14)$ \\
\hline $\mathrm{N} 4-\mathrm{C} 7-\mathrm{N} 3$ & $114.60(13)$ \\
\hline $\mathrm{N} 5-\mathrm{C} 8-\mathrm{C} 10$ & $119.06(14)$ \\
\hline $\mathrm{N} 5-\mathrm{C} 8-\mathrm{C} 9$ & $124.62(14)$ \\
\hline $\mathrm{C} 10-\mathrm{C} 8-\mathrm{C} 9$ & $116.31(13)$ \\
\hline $\mathrm{N} 3-\mathrm{C} 9-\mathrm{C} 8$ & $112.26(12)$ \\
\hline N3-C9-H9A & 109.2 \\
\hline $\mathrm{C} 8-\mathrm{C} 9-\mathrm{H} 9 \mathrm{~A}$ & 109.2 \\
\hline $\mathrm{C} 6-\mathrm{N} 2-\mathrm{N} 3-\mathrm{C} 7$ & $177.92(15)$ \\
\hline $\mathrm{C} 6-\mathrm{N} 2-\mathrm{N} 3-\mathrm{C} 9$ & $-6.1(2)$ \\
\hline $\mathrm{C} 7-\mathrm{N} 4-\mathrm{N} 5-\mathrm{C} 8$ & $4.1(2)$ \\
\hline $\mathrm{C} 16-\mathrm{N} 7-\mathrm{N} 8-\mathrm{C} 17$ & $172.24(12)$ \\
\hline $\mathrm{C} 16-\mathrm{N} 7-\mathrm{N} 8-\mathrm{C} 19$ & $-6.07(19)$ \\
\hline $\mathrm{C} 17-\mathrm{N} 9-\mathrm{N} 10-\mathrm{C} 18$ & $4.7(2)$ \\
\hline $\mathrm{C} 5-\mathrm{N} 1-\mathrm{C} 1-\mathrm{C} 2$ & $-1.8(3)$ \\
\hline $\mathrm{N} 1-\mathrm{C} 1-\mathrm{C} 2-\mathrm{C} 3$ & $1.3(3)$ \\
\hline $\mathrm{C} 1-\mathrm{C} 2-\mathrm{C} 3-\mathrm{C} 4$ & $0.8(3)$ \\
\hline $\mathrm{C} 2-\mathrm{C} 3-\mathrm{C} 4-\mathrm{C} 5$ & $-2.1(2)$ \\
\hline $\mathrm{C} 2-\mathrm{C} 3-\mathrm{C} 4-\mathrm{C} 6$ & $178.24(16)$ \\
\hline $\mathrm{C} 1-\mathrm{N} 1-\mathrm{C} 5-\mathrm{C} 4$ & $0.3(3)$ \\
\hline $\mathrm{C} 3-\mathrm{C} 4-\mathrm{C} 5-\mathrm{N} 1$ & $1.7(3)$ \\
\hline $\mathrm{C} 6-\mathrm{C} 4-\mathrm{C} 5-\mathrm{N} 1$ & $-178.69(16)$ \\
\hline $\mathrm{N} 3-\mathrm{N} 2-\mathrm{C} 6-\mathrm{C} 4$ & $179.19(14)$ \\
\hline $\mathrm{C} 5-\mathrm{C} 4-\mathrm{C} 6-\mathrm{N} 2$ & $174.00(15)$ \\
\hline $\mathrm{C} 3-\mathrm{C} 4-\mathrm{C} 6-\mathrm{N} 2$ & $-6.4(3)$ \\
\hline $\mathrm{N} 5-\mathrm{N} 4-\mathrm{C} 7-\mathrm{O} 1$ & $177.59(16)$ \\
\hline $\mathrm{N} 5-\mathrm{N} 4-\mathrm{C} 7-\mathrm{N} 3$ & $-3.9(2)$ \\
\hline $\mathrm{N} 2-\mathrm{N} 3-\mathrm{C} 7-\mathrm{O} 1$ & $-4.7(2)$ \\
\hline $\mathrm{C} 9-\mathrm{N} 3-\mathrm{C} 7-\mathrm{O} 1$ & $179.44(15)$ \\
\hline $\mathrm{N} 2-\mathrm{N} 3-\mathrm{C} 7-\mathrm{N} 4$ & $176.77(13)$ \\
\hline $\mathrm{C} 9-\mathrm{N} 3-\mathrm{C} 7-\mathrm{N} 4$ & $1.0(2)$ \\
\hline $\mathrm{N} 4-\mathrm{N} 5-\mathrm{C} 8-\mathrm{C} 10$ & $179.51(15)$ \\
\hline $\mathrm{N} 4-\mathrm{N} 5-\mathrm{C} 8-\mathrm{C} 9$ & $-1.3(2)$ \\
\hline $\mathrm{N} 2-\mathrm{N} 3-\mathrm{C} 9-\mathrm{C} 8$ & $-174.34(14)$ \\
\hline
\end{tabular}

$\begin{array}{ll}\text { O2-C17-N9 } & 121.57(14) \\ \text { O2-C17-N8 } & 123.36(14) \\ \text { N9-C17-N8 } & 115.07(12) \\ \text { N10-C18-C20 } & 118.78(14) \\ \text { N10-C18-C19 } & 125.12(13) \\ \text { C20-C18-C19 } & 116.10(14) \\ \text { N8-C19-C18 } & 112.06(12) \\ \text { N8-C19-H19A } & 109.2 \\ \text { C18-C19-H19A } & 109.2 \\ \text { N8-C19-H19B } & 109.2 \\ \text { C18-C19-H19B } & 109.2 \\ \text { H19A-C19-H19B } & 107.9 \\ \text { C18-C20-H20A } & 109.5 \\ \text { C18-C20-H20B } & 109.5 \\ \text { H20A-C20-H20B } & 109.5 \\ \text { C18-C20-H20C } & 109.5 \\ \text { H20A-C20-H20C } & 109.5 \\ \text { H20B-C20-H20C } & 109.5 \\ \text { C7-N3-C9-C8 } & \\ \text { N5-C8-C9-N3 } & 1.3(2) \\ \text { C10-C8-C9-N3 } & -1.1(2) \\ \text { C15-N6-C11-C12 } & 178.12(14) \\ \text { N6-C11-C12-C13 } & 0.4(2) \\ \text { C11-C12-C13-C14 } & 1.4(3) \\ \text { C12-C13-C14-C15 } & -1.7(2) \\ \text { C12-C13-C14-C16 } & 0.2(2) \\ \text { C11-N6-C15-C14 } & 179.96(14) \\ \text { C13-C14-C15-N6 } & -2.0(2) \\ \text { C16-C14-C15-N6 } & 1.7(2) \\ \text { N8-N7-C16-C14 } & -178.07(14) \\ \text { C13-C14-C16-N7 } & 179.00(12) \\ \text { C15-C14-C16-N7 } & -8.9(2) \\ \text { N10-N9-C17-O2 } & 170.84(14) \\ \text { N10-N9-C17-N8 } & 174.79(13) \\ \text { N7-N8-C17-O2 } & -5.0(2) \\ \text { C19-N8-C17-O2 } & 1.8(2) \\ \text { N7-N8-C17-N9 } & -179.92(13) \\ \text { C19-N8-C17-N9 } & -178.42(11) \\ \text { N9-N10-C18-C20 } & -0.15(19) \\ \text { N9-N10-C18-C19 } & -178.69(13) \\ \text { N7-N8-C19-C18 } & 0.8(2) \\ \text { C17-N8-C19-C18 } & -177.33(11) \\ \text { N10-C18-C19-N8 } & 4.49(19) \\ \text { C20-C18-C19-N8 } & -5.0(2) \\ & 174.58(12)\end{array}$




\section{supporting information}

Hydrogen-bond geometry $\left(\AA,{ }^{\circ}\right)$

\begin{tabular}{lllll}
\hline$D-\mathrm{H} \cdots A$ & $D-\mathrm{H}$ & $\mathrm{H} \cdots A$ & $D \cdots A$ & $D-\mathrm{H} \cdots A$ \\
\hline $\mathrm{N} 4-\mathrm{H} 4 \cdots \mathrm{O} 2^{\mathrm{i}}$ & 0.88 & 2.32 & $2.9545(17)$ & 129 \\
$\mathrm{~N} 4-\mathrm{H} 4 \cdots \mathrm{N}^{\mathrm{i}}$ & 0.88 & 2.43 & $3.2346(17)$ & 152 \\
$\mathrm{~N} 9-\mathrm{H} 9 \cdots \mathrm{N} 66^{\mathrm{ii}}$ & 0.88 & 2.04 & $2.882(2)$ & 159 \\
$\mathrm{C} 19-\mathrm{H} 19 A \cdots \mathrm{N} 1^{\mathrm{ii}}$ & 0.99 & 2.57 & $3.0852(19)$ & 112 \\
$\mathrm{C} 19-\mathrm{H} 19 A \cdots{ }^{\mathrm{ii}}$ & 0.99 & 2.60 & $3.5596(19)$ & 164 \\
$\mathrm{C} 20-\mathrm{H} 20 C \cdots \mathrm{O} 1^{\mathrm{v}}$ & 0.98 & 2.53 & $3.3906(19)$ & 147
\end{tabular}

Symmetry codes: (i) $x-1 / 2,-y+1 / 2, z+1 / 2$; (ii) $x, y, z-1$; (iii) $x-1, y, z-1$; (iv) $x-1 / 2,-y+1 / 2, z-1 / 2$; (v) $-x+1 / 2, y-1 / 2,-z+1 / 2$. 